Nervenarzt 2021 · 92:744-751

https://doi.org/10.1007/s00115-021-01122-z

Angenommen: 6. April 2021

Online publiziert: 3. Mai 2021

(c) Springer Medizin Verlag $\mathrm{GmbH}$, ein Teil von Springer Nature 2021

\author{
Steffen Tiedt ${ }^{1} \cdot$ Franziska Dorn ${ }^{2}$ \\ ' Institut für Schlaganfall- und Demenzforschung, Klinikum der Universität München, LMU München, \\ München, Deutschland \\ ${ }^{2}$ Klinik für Neuroradiologie, Universitätsklinikum Bonn, Bonn, Deutschland
}

\title{
Thrombektomie in der klinischen Praxis - Was lernen wir aus Registerstudien?
}

\begin{abstract}
Randomisierte Studien sind für die Etablierung neuer Behandlungen essenziell, die Ergebnisse aber meist nicht unmittelbar in die klinische Praxis übertragbar. Registerstudien bilden die Versorgungsrealität besser ab und helfen deshalb dabei, Behandlungseffekte außerhalb kontrollierter Studienbedingungen und in Untergruppen darzustellen sowie technische Entwicklungen zu überprüfen. In der vorliegenden Arbeit zeigen wir, dass Registerstudien eine große Bedeutung bei der Bewertung der mechanischen Thrombektomie haben und Informationen über randomisierte Studien hinaus liefern können.
\end{abstract}

\section{Hintergrund}

Die Veröffentlichung von fünf randomisiert kontrollierten Studien (RCTs) zur endovaskulären Thrombektomie proximaler Gefäßverschlüsse der vorderen Strombahn änderte die klinische Praxis 2015 schlagartig [1, 2, 5, 9, 14]. Alle Studien belegten die hohe Wirksamkeit der Thrombektomie in der akuten Schlaganfallbehandlung, vier der Studien wurden nach Überlegenheitsnachweis in Interimsanalysen vorzeitig abgebrochen. Im Vergleich mit vorherigen RCTs, die keine Überlegenheit der endovaskulären Therapie nachweisen konnten, wurde dieser Erfolg neben dem Einsatz von Stent-Retrievern auch den stringenteren Ein- und Ausschlusskriterien zugeschrieben. Eingeschlossen wurden vor allem Patienten, die 1. einen akuten Verschluss der intrakraniellen A. carotis interna oder des ersten Segments der A. cerebri media aufwiesen, 2. mit einer akut mittel- bis schwergradig ausgeprägten Symptomatik bei 3. sehr gutem funktionellem Ausgangsniveau präsentierten und 4 . in einem frühen Zeitfenster behandelt werden konnten $(<6 \mathrm{~h})$. Für diese Patientenpopulation betrug die „number needed to treat" mittels Thrombektomie für eine Verbesserung des funktionellen Ergebnisses nach 90 Tagen (ein Punkt auf der modifizierten Rankin-Skala [mRS]) in den RCTs 2,6 [6]. Leitlinien sprechen dementsprechend eine starke Empfehlung für die mechanische Thrombektomie in dieser Patientengruppe aus [13, 18], sodass die Thrombektomie seit 2015 flächendeckend in die klinische Routine implementiert wurde.

\section{RCT vs. prospektive Register- studie}

Warum ist es notwendig, Daten aus der klinischen Praxis, sog. Real-world-Daten, in Registerstudien zu erheben, wenn die Wirksamkeit der Therapie in mehreren RCTs gezeigt werden konnte? Registerstudien bilden die Patientenversorgung außerhalb kontrollierter Studienbedingungen $\mathrm{ab}$ und liefern damit wertvolle Daten zu Abläufen in der klinischen Routine. Prospektive Registerstudien mit Erfassung des Langzeitoutcomes stellen dabei die höchste Qualitätsstufe von Real-world-Daten dar [12] und sind deshalb auch für die Thrombektomie ein häufig genutztes Mittel, um u. a. die Ver- sorgung in einzelnen Ländern oder mit spezifischen Devices und Techniken zu untersuchen (•Tab. 1). Im Vergleich zu RCTs sind prospektive Registerstudien administrativ leichter und kostengünstiger umsetzbar, sind deshalb idealerweise nicht auf ein Sponsoring angewiesen und erlauben aufgrund inklusiverer Einschlusskriterien eine schnellere Patientenrekrutierung (•Tab. 2). Die Mehrzahl der Register konnte über kürzere Rekrutierungszeiträume tatsächlich mehr Patienten einschließen als die Thrombektomie-Gruppen der fünf genannten RCTs zusammen ( $n=634$, • Tab. 1 ; [6]). Grundsätzlich können folgende Registerstudien unterschieden werden:

- länderspezifische Register, in die die Daten mit Thrombektomie behandelter Patienten eines Landes eingehen, z. B. in den Niederlanden das MR CLEAN Register (Multicenter collaboration for endovascular treatment of acute ischemic stroke in the Netherlands Registry; [8]), in Frankreich das ETIS-Register (Endovascular Treatment in Ischemic Stroke; [3]) und in Deutschland das GSR-ET (German Stroke Registry Endovascular Treatment; [19]),

- Device-basierte Studien, in denen die Sicherheit und Wirksamkeit eines einzelnen Devices untersucht wird und die in der Regel gesponsert sind sowie restriktivere Ein- und Ausschlusskriterien haben (• Tab. 1). 
Hier steht eine Anzeige.

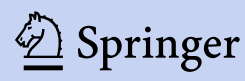




\section{Leitthema}

Tab. 1 Multizentrische Registerstudien für Thrombektomie

\begin{tabular}{|c|c|c|c|c|c|}
\hline Registerstudie & $n$ & Rekrutierungszeit & Land & Zentren & Einschlusskriterien $^{\mathrm{a}}$ \\
\hline \multicolumn{6}{|l|}{ Länderspezifisch } \\
\hline GSR-ET & 6635 & 06/2015 - „ongoing“ & $\mathrm{DE}$ & 25 & - \\
\hline \multirow[t]{3}{*}{ MR CLEAN-R ${ }^{b}$} & \multirow[t]{3}{*}{3615} & \multirow[t]{3}{*}{ 04/2014 - „ongoing“ } & \multirow[t]{3}{*}{$\mathrm{NL}$} & \multirow[t]{3}{*}{17} & Anteriore Zirkulation \\
\hline & & & & & $\mathrm{T} \rightarrow$ Punktion $\leq 6,5 \mathrm{~h}$ \\
\hline & & & & & $\mathrm{pmRS} \leq 2$ \\
\hline \multirow[t]{3}{*}{ Italian Reg. of EVT } & \multirow[t]{3}{*}{3057} & \multirow[t]{3}{*}{ 01/2011 - „ongoing" } & \multirow[t]{3}{*}{ IT } & \multirow[t]{3}{*}{25} & Anteriore Zirkulation \\
\hline & & & & & $\mathrm{T} \rightarrow$ Aufnahme $\leq 6 \mathrm{~h}$ \\
\hline & & & & & $\mathrm{pmRS} \leq 2$ \\
\hline ETIS & 1986 & $05 / 2012-01 / 2019$ & $\mathrm{FR}$ & 6 & - \\
\hline \multirow{2}{*}{ RESCUE 2} & \multirow[t]{2}{*}{1932} & \multirow[t]{2}{*}{$10 / 2014-09 / 2016$} & \multirow[t]{2}{*}{$J P$} & \multirow[t]{2}{*}{46} & Alter $\geq 20$ Jahre \\
\hline & & & & & $\mathrm{T} \rightarrow$ Aufnahme $\leq 24 \mathrm{~h}$ \\
\hline ANGEL & 741 & $06 / 2015-12 / 2017$ & $\mathrm{CN}$ & 20 & NIHSS-Score $\geq 6$ \\
\hline \multirow[t]{4}{*}{ ACTUAL } & \multirow[t]{4}{*}{698} & \multirow[t]{4}{*}{$01 / 2014-06 / 2016$} & \multirow[t]{4}{*}{$\mathrm{CN}$} & \multirow[t]{4}{*}{21} & ASPECTS $\geq 6$ \\
\hline & & & & & $\mathrm{T} \rightarrow$ Punktion $\leq 6 \mathrm{~h}$ \\
\hline & & & & & NIHSS Score $\geq 6$ \\
\hline & & & & & $\mathrm{pmRS} \leq 1$ \\
\hline \multirow{3}{*}{ SELECT } & \multirow[t]{3}{*}{361} & \multirow[t]{3}{*}{$01 / 2016-02 / 2018$} & \multirow[t]{3}{*}{ US } & \multirow[t]{3}{*}{9} & Anteriore Zirkulation \\
\hline & & & & & $\mathrm{T} \rightarrow$ Punktion $\leq 8 \mathrm{~h}$ \\
\hline & & & & & $\mathrm{pmRS} \leq 1$ \\
\hline \multicolumn{6}{|l|}{ Device-basiert } \\
\hline BEYOND-SWIFT & 2397 & $11 / 2017-12 / 2018$ & 5 Länder & 7 & Medtronic-Stent-Retriever \\
\hline TREVO 2000 & 2008 & $11 / 2013-05 / 2017$ & 12 Länder & 76 & Trevo-Stent-Retriever \\
\hline \multirow[t]{4}{*}{ STRATIS } & 1000 & $08 / 2014-06 / 2016$ & US & 55 & Medtronic-Stent-Retriever \\
\hline & & & & & $\mathrm{T} \rightarrow$ Punktion $\leq 8 \mathrm{~h}$ \\
\hline & & & & & $\mathrm{pmRS} \leq 1$ \\
\hline & & & & & NIHSS-Score $\geq 8, \leq 30$ \\
\hline TRACK & 634 & $03 / 2013-08 / 2015$ & US & 23 & Trevo-Stent-Retriever \\
\hline NASA & 354 & $02 / 2012-02 / 2013$ & US & 24 & Solitaire FR-Stent-Retriever \\
\hline ARISE II & 227 & $10 / 2015-02 / 2017$ & 8 Länder & 19 & Embotrap II \\
\hline & & & & & $\mathrm{T} \rightarrow$ Punktion $\leq 8 \mathrm{~h}$ \\
\hline & & & & & $\mathrm{pmRS} \leq 1$ \\
\hline & & & & & NIHSS-Score $\geq 8, \leq 25$ \\
\hline STAR & 202 & $10 / 2010-02 / 2012$ & 6 Länder & 14 & Solitaire FR-Stent-Retriever \\
\hline & & & & & Anteriore Zirkulation \\
\hline & & & & & $\mathrm{pmRS} \leq 2$ \\
\hline & & & & & NIHSS-Score $\geq 8, \leq 30$ \\
\hline
\end{tabular}

ACTUAL Endovascular Treatment for Acute Anterior Circulation Ischemic Stroke Registry, ANGEL Acute Ischemic stroke Cooperation Group of Endovascular Treatment, ASPECTS Alberta Stroke Program Early Computed Tomography Score, BEYOND-SWIFT Bernese-European Registry for Ischemic Stroke Patients Treated Outside Current Guidelines With Neurothrombectomy Devices Using the Solitaire FR With the Intention for Thrombectomy, CN China, DE Deutschland, ETIS Endovascular Treatment in Ischemic Stroke, EVT endovascular treatment, FR Frankreich, GSR-ET German Stroke Registry - Endovascular Treatment, InkI Einschlusskriterien, IT Italien, JP Japan, MR CLEAN-R Multicenter collaboration for endovascular treatment of acute ischemic stroke in the Netherlands Registry, NL Niederlande, pmRS prämorbide modifizierte Rankin-Skala, Reg. „registry“, RESCUE Recovery by Endovascular Salvage for Cerebral Ultra-Acute Embolism (RESCUE) Japan Registry 2, SELECT Optimizing Patient Selection for Endovascular Treatment in Acute Ischemic Stroke, STAR Solitaire Flow Restoration Thrombectomy for Acute Revascularization, STRATIS Systematic Evaluation of Patients Treated with Neurothrombectomy Devices for Acute Ischemic Stroke, TRACK TREVO Stent-Retriever Acute Stroke, NASA North American Solitaire Stent Retriever Acute Stroke, US USA

${ }^{2}$ Kriterien für Patienteinschluss über: 1. akuter ischämischer Schlaganfall, 2. Verschluss eines proximalen Gefäßes, 3. Initiierung der Thrombektomie und 4. Alter $\geq 18$ Jahre hinaus

${ }^{b}$ Kerndatensatz 


\section{Aus Registerstudien lernen}

Große Registerstudien konnten in den letzten Jahren zeigen, dass die endovaskuläre Thrombektomie in der klinischen Praxis sicher und effektiv eingesetzt wird, sich die Ergebnisse randomisierter Studien aber nicht unverändert in die klinische Routine übertragen lassen (• Tab. 3).

Die niederländische MR-CLEANRegistry-Studie startete unmittelbar nach Abschluss der randomisierten MRCLEAN-Studie mit der prospektiven Datenerhebung aller endovaskulär behandelten Schlaganfallpatienten an 16 niederländischen Zentren. In einer Analyse von 1488 Patienten mit Verschlüssen der vorderen Zirkulation, die innerhalb von $6,5 \mathrm{~h}$ nach Symptombeginn behandelt wurden, zeigten signifikant mehr Patienten ein gutes funktionelles Ergebnis nach 90 Tagen als in der randomisierten MR-CLEAN-Studie (38\% vs. $33 \%$ ). Die Patienten des Registers waren dabei sogar durchschnittlich älter und hatten eine höhere Prävalenz von Komorbiditäten, wurden jedoch im Vergleich zu Patienten aus der randomisierten Studie etwa $1 \mathrm{~h}$ schneller behandelt [8].

\section{》) Registerdaten erfassen Patientengruppen, die in RCTs unterrepräsentiert sind}

In der deutschen GSR-ET-Studie wurden zwischen Juni 2015 und Dezember 2019 an 25 Zentren insgesamt 6635 mit Thrombektomie behandelte Patienten erfasst. Trotz niedrigerer Symptomschwere bei Aufnahme im Vergleich zu den RCTs (National Institutes of Health Stroke Scale [NIHSS]-Score Median 15 vs. 17) und im Mittel schnellerer und technisch erfolgreicherer Behandlung war ein gutes funktionelles Ergebnis nach 90 Tagen seltener ( $37 \%$ vs. $46 \%$ ). Im Vergleich zur RCT-Kontrollgruppe ohne Thrombektomie (mRS 0-2 nach 90 Tagen: 27\%; [6]) ist das klinische Ergebnis in der GSR-ETStudie zwar besser, bedeutet aber praktisch fast eine Halbierung des Behandlungseffekts der RCTs. Dies könnte vor allem in den unterschiedlichen Patientenpopulationen begründet liegen: Die Patienten der GSR-ET-Studie waren im

Nervenarzt 2021 -92:744-751 https://doi.org/10.1007/s00115-021-01122-z

๑) Springer Medizin Verlag GmbH, ein Teil von Springer Nature 2021

\section{S. Tiedt $\cdot$ F. Dorn}

\section{Thrombektomie in der klinischen Praxis - Was lernen wir aus Registerstudien?}

\section{Zusammenfassung}

Hintergrund. Fünf randomisiert kontrollierte Studien (RCTs) konnten 2015 die Wirksamkeit der Thrombektomie bei selektierten Schlaganfallpatienten mit akuten proximalen Gefäßverschlüssen der vorderen Strombahn belegen und führten zur flächendeckenden Implementierung der Thrombektomie in die klinische Praxis.

Ziel der Arbeit. Es soll der Mehrwert, der sich über RCTs hinaus aus der registerbasierten Erfassung von Daten aus der klinischen Praxis ergibt, dargestellt werden.

Material und Methoden. Durch eine Literaturreview wurden RCTs und Registerstudien identifiziert und ihre Ergebnisse, wenn möglich, in Relation zueinander gesetzt. Ergebnisse. Daten aus Thrombektomieregisterstudien sind wichtig um 1 . die Übertragung der Ergebnisse aus RCTs in die klinische Praxis beurteilen zu können, 2. die Wirksamkeit der Thrombektomie in Patientengruppen, die in randomisierten Studien unterrepräsentiert waren, z. B. ältere Patienten, zu beschreiben, 3 . die Performance von Devices vergleichen oder technische Weiterentwicklungen beurteilen zu können sowie 4. die Versorgungsqualität über die Zeit darzustellen.

Diskussion. Über RCTs hinaus sind Registerstudien von großer Bedeutung für die kontinuierliche Analyse und Verbesserung von Behandlungsprozessen sowie technischer Entwicklungen.

Schlüsselwörter

Schlaganfall · Versorgungsrealität · Endovaskuläre Therapie - Randomisiert kontrollierte Studien · Technische Entwicklungen

\section{Thrombectomy in clinical practice-What do we learn from registry studies?}

\section{Abstract}

Background. In 2015, randomized controlled trials (RCT) provided high-level evidence for the efficacy of endovascular thrombectomy in selected patients with acute ischemic stroke due to large vessel occlusion of the anterior circulation. Ever since, thrombectomy is strongly recommended for these patients and has been broadly implemented in clinical practice.

Objective. To determine whether registry studies depicting real-life data provide additional information beyond RCTs. Material and methods. Literature review of RCTs and registry studies related to thrombectomy.

Results. Data from registry studies on thrombectomy are important to 1 . evaluate whether $\mathrm{RCT}$ results can be directly transferred into clinical practice, 2. comparatively describe the efficacy of thrombectomy in patient groups underrepresented in RCTs, such as older patients, 3. compare device performances and assess technical developments, and 4. determine how treatment processes and outcomes change over time.

Conclusion. Beyond RCTs, registry studies are imperative for the continuous analysis and improvement of treatment processes and outcomes as well as technical devices in daily clinical practice.

Keywords

Stroke - Real-world data - Endovascular treatment $\cdot$ Randomized controlled studies . Technical developments
Median 8 Jahre älter als in den RCTs, hatten häufiger vaskuläre Risikofaktoren und zeigten 4-mal so häufig schon vor dem Schlaganfall eine funktionelle Einschränkung (•Tab. 3).

Registerdaten helfen die Ergebnisse randomisiert kontrollierter Studien besser einordnen zu können und zukünftige RCTs in Bezug auf Zielsetzung, Design und Fallzahlkalkulation zu informieren [16]. So können Registerdatensätze Patientengruppen identifizieren, die möglicherweise von der Therapie profitieren, in den initialen RCTs aber unterrepräsentiert oder sogar ausgeschlossen waren; speziell auf diese Patientengruppen könnten zukünftige RCTs ausgerichtet werden, um die Evidenz zu stärken. Die 
Tab. 2 Charakteristika von randomisiert kontrollierten Studien und Registerstudien

\begin{tabular}{|c|c|c|}
\hline & RCT & Registerstudien \\
\hline Datenquelle & $\begin{array}{l}\text { Hochstrukturierte Forschungsumge- } \\
\text { bung an spezialisierten Zentren }\end{array}$ & $\begin{array}{l}\text { Alltägliche klinische Praxis an kleinen } \\
\text { und großen Zentren }\end{array}$ \\
\hline $\begin{array}{l}\text { Ein-/Ausschluss- } \\
\text { kriterien }\end{array}$ & Meist streng & Inklusiv \\
\hline Population & Hochselektiert, klein & Breite Masse, groß \\
\hline Validität & Intern & Extern \\
\hline Bias & Unwahrscheinlich & Wahrscheinlich \\
\hline Confounding & Minimal & Vorhanden \\
\hline Kosten & Hoch & Niedrig \\
\hline
\end{tabular}

Tab. 3 Patientencharakteristika in randomisiert kontrollierten Studien und Registerstudien

\begin{tabular}{|c|c|c|}
\hline & $\begin{array}{l}\text { HERMES } \\
n=634\end{array}$ & $\begin{array}{l}\text { GSR } \\
n=6635\end{array}$ \\
\hline \multicolumn{3}{|l|}{ Demographische Parameter } \\
\hline Alter, Median (IQR) [Jahre] & $68(57-77)$ & $76(65-82)$ \\
\hline Weiblich & $48 \%$ & $51 \%$ \\
\hline \multicolumn{3}{|l|}{ Komorbiditäten und Vorzustand } \\
\hline Bluthochdruck & $56 \%$ & $78 \%$ \\
\hline Diabetes Mellitus & $13 \%$ & $22 \%$ \\
\hline Vorhofflimmern & $33 \%$ & $42 \%$ \\
\hline Prämorbide mRS > 1 & $5 \%$ & $21 \%$ \\
\hline \multicolumn{3}{|l|}{ Baseline nach Schlaganfall } \\
\hline M1-Verschluss & $69 \%$ & $58 \%$ \\
\hline ASPECTS, Median (IQR)/0-5 [\%] & $9(7-10) / 9$ & $9(7-10) / 9$ \\
\hline NIHSS-Score, Median (IQR) & $17(14-20)$ & $15(9-19)$ \\
\hline \multicolumn{3}{|l|}{ Behandlung } \\
\hline Intravenöse Thrombolyse & $83 \%$ & $51 \%$ \\
\hline $\mathrm{T}$ (Onset $\rightarrow$ Groin), Median (IQR) [min] & $239(194-299)$ & $195(140-275)$ \\
\hline $\mathrm{mTICl} 2 \mathrm{~b} / 3$ & $71 \%$ & $85 \%$ \\
\hline \multicolumn{3}{|l|}{ Outcome } \\
\hline 24 h-NIHSS-Score, Median (IQR) & $8(3-16)$ & $10(4-19)$ \\
\hline mRS 0-2 nach 90 Tagen & $46 \%$ & $37 \%$ \\
\hline \multicolumn{3}{|c|}{$\begin{array}{l}\text { HERMES-Daten entsprechend Goyal et al. } 2016 \text { [6] } \\
\text { ASPECTS Alberta Stroke Program Early CT Score, GSR German Stroke Registry, HERMES Highly Effective } \\
\text { Reperfusion evaluated in Multiple Endovascular Stroke Trials, IQR Interquartilenabstand, mRS mo- } \\
\text { difizierte Rankin-Skala, } m \text { TICI modified Thrombolysis in Cerebral Infarction, M1 erstes Segment der } \\
\text { A. cerebri media, NIHSS National Institutes of Health Stroke Scale, TZeit }\end{array}$} \\
\hline
\end{tabular}

Datenlage für einige dieser Patientengruppen, z.B. solche mit distalen Verschlüssen oder niedrigem NIHSS-Score, wird in der Arbeit von Leo Bonati und Marios Psychogios in dieser Ausgabe von Der Nervenarzt dargestellt.

Für andere Patientengruppen erscheint die Durchführung dezidierter RCTs weniger sinnvoll, sodass Registerdaten eine hohe Relevanz bei der Beschreibung von Therapieeffekten und damit für Empfehlungen in Leitlinien besitzen. Tatsächlich weisen nur 8-14\%
RCTs erfolgt - nicht prinzipiell von der Therapie ausgeschlossen werden sollten [3]. In der MR-CLEAN-Registry-Studie hatten Patienten über 80 Jahre zwar insgesamt ein schlechteres Ergebnis als jüngere Patienten, der relative Benefit einer erfolgreichen Reperfusion war in dieser Patientengruppe aber größer [7].

Wie stark klinische Variablen (z. B. die seit Symptombeginn vergangene Zeit) das funktionelle Ergebnis nach einer Thrombektomie beeinflussen, ist von hoher Bedeutung für individuelle Therapieentscheidungen. RCT- und registerbasierte Analysen zeigen, dass die Wahrscheinlichkeit für ein gutes Outcome mit zunehmender Zeit seit Symptombeginn abnimmt [11, 15]. Während Sekundäranalysen aus RCTs in diesen Studien zur Identifizierung und Gewichtung dieser Outcomeprädiktoren den Vorteil der Randomisierung verlieren, da sich die Analysen auf die Interventionsgruppe beschränken, bieten Registerdaten eine breitere Patientenpopulation mit stärkerer Vertretung von Randgruppen wie z. B. älteren Patienten und zeigen deshalb oft andere Effekte: Während die Wahrscheinlichkeit für ein gutes funktionelles Outcome mit jeder Stunde Verspätung in RCT-Daten um 5,2\% sank [15], lag diese Zahl in Registerdaten bei 7,7\% und war damit etwa $50 \%$ höher [11].

\section{》) Mit Registerstudien lassen sich technische Weiterentwicklungen analysieren}

Endovaskuläre Techniken entwickeln sich stetig weiter. Die kontinuierliche Überprüfung der Wirksamkeit neuer Devices sowie weiterentwickelter vorhandener Systeme durch randomisierte Studien wäre - bei vorhandener Evidenz für die Methode an sich - nicht praktikabel. Prospektive Registerstudien bieten deshalb das höchste erreichbare Evidenzlevel, um diese Entwicklungen analysieren zu können. Die Performance verschiedener Stent-Retriever wurde innerhalb definierter Ein- und Ausschlusskriterien in unterschiedlichen Studien untersucht und kann so in Rela- 
Hier steht eine Anzeige.

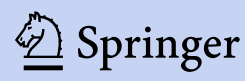


tion gesetzt werden: Der Prozentsatz für eine erfolgreiche Rekanalisation (modified Thrombolysis in Cerebral Infarction [mTICI] Score 2b/3) in großen industriegesponserten Registerstudien war dabei im Allgemeinen höher als in den RCTs (80\% in TRACK [22], $88 \%$ in STRATIS [10] und NASA [21], $93 \%$ in ARISE II [20] vs. $71 \%$ in HERMES [6]). Der prozentuale Anteil an Patienten mit einem guten funktionellen Outcome lag mindestens auf RCT-Niveau ( $42 \%$ in NASA, $48 \%$ in TRACK, $57 \%$ in STRATIS und $67 \%$ in ARISE II vs. $46 \%$ in HERMES) und damit höher als in Registerstudien ohne entsprechende Einschlusskriterien (MR CLEAN Registry 38\%, GSR-ET $37 \%)$.

Darüber hinaus erlauben Registerdaten die Analyse spezieller technischer Aspekte: Mittels Daten aus STRATIS, NASA und TRACK konnte gezeigt werden, dass die Verwendung eines Ballonkatheters unabhängig vom verwendeten Stent-Retriever (Solitaire FR und Trevo) ein unabhängiger Prädiktor für ein gutes klinisches Ergebnis ist [21-23]. In NASADaten war darüber hinaus die Verwendung längerer Stent-Retriever häufiger mit einer erfolgreichen Rekanalisation und einer First-pass-Rekanalisation assoziiert [23].

Nicht zuletzt ermöglichen fortlaufende Registerdaten die vergleichende Darstellung der Versorgungsqualität über die Zeit. So können nicht nur landesweite und zentrumsspezifische Entwicklungen verschiedener Qualitätsmerkmale intern beurteilt werden, sondern auch der Einfluss äußerer Faktoren, wie z. B. die COVID-19-Pandemie, auf die Versorgung von Thrombektomiepatienten analysiert werden. Konkret konnte so mittels der Daten aus dem GSR-ET gezeigt werden, dass in Deutschland zwar die Gesamtzahl der behandelten Schlaganfallpatienten während der ersten Pandemiewelle im Vergleich zum Vorjahreszeitraum abnahm, jedoch Anzahl und Prozesszeiten der Thrombektomien sowie Outcome der behandelten Patienten im Wesentlichen stabil blieben [17].

\section{Limitationen}

Durch das Fehlen einer Randomisierung und einer Kontrollgruppe ohne Behandlung sind Registerstudien anfällig für systematische Verzerrungen (Bias) sowie Störvariablen („confounding“). Eine besondere Gefahr stellt das durch den unkontrollierten Ein- oder Ausschluss von Patienten in das Register hervorgerufene Selektionsbias dar, dem z. B. durch eine prädefinierte minimale Einschlussrate pro Zentrum vorgebeugt werden kann. Neuere methodische Entwicklungen in der statistischen Analyse wie das Matchen mittels Propensity Scores, Bootstrapping und Algorithmen des maschinellen Lernens bieten zudem die Möglichkeit, die Effekte von Verzerrungen und Störvariablen nach Erhebung der Daten abzuschwächen [12]. Durch das Fehlen eines unabhängigen externen Monitorings und einer zentralen Bilddatenauswertung können Registerstudien im Vergleich zu RCTs eine geringere Datenvollständigkeit und -qualität zeigen.

\section{Fazit für die Praxis}

- Registerstudien stellen dar, wie sich Ergebnisse aus RCTs in die klinische Praxis übertragen lassen: $\mathbf{3 7} \%$ der mit Thrombektomie behandelten Patienten zeigten in deutschen Registerdaten ein gutes funktionelles Outcome im Vergleich zu $27 \%$ in der RCT-Kontroll- und $46 \%$ in der Behandlungsgruppe.

- Ergebnisse aus Registerstudien stellen die Grundlage für die Mehrheit der Empfehlungen in Leitlinien dar und sind von besonderer Bedeutung für Patientengruppen, für die eine dezidierte RCT unwahrscheinlich ist: z. B. zeigten Registerdaten, dass die Thrombektomie auch bei älteren Patienten sicher und wirksam ist. - Registerstudien ermöglichen den Vergleich von Performance-Indikatoren zwischen Devices oder nach technischen Weiterentwicklungen sowie die vergleichende Darstellung der Versorgungsqualität über die Zeit, z. B. auch während der COVID19-Pandemie: Daten aus dem deut- schen Schlaganfallregister fanden keinen Einfluss der Pandemie auf die Prozesszahlen und -zeiten sowie das funktionelle Ergebnis nach Thrombektomie.

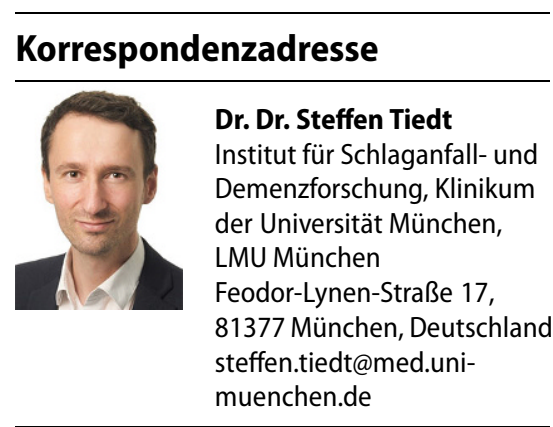

\section{Einhaltung ethischer Richtlinien}

Interessenkonflikt. S. Tiedt erhält Unterstützung für Forschungsvorhaben von der Corona-Stiftung. F. Dorn hat finanzielle Unterstützung für Forschungsprojekte von Cerenovus, finanzielle Gegenleistungen für Berater- und Proctortätigkeiten von Cerus, Balt Germany und Phenox sowie Vortragshonorare von Acandis und Cerenovus erhalten.

Für diesen Beitrag wurden von den Autoren keine Studien an Menschen oder Tieren durchgeführt. Für die aufgeführten Studien gelten die jeweils dort angegebenen ethischen Richtlinien.

\section{Literatur}

1. Berkhemer OA, Fransen PS, Beumer D et al (2015) A randomized trial of intraarterial treatment for acute ischemic stroke. N Engl J Med 372:11-20

2. Campbell BC, Mitchell PJ, Kleinig TJ et al (2015) Endovascular therapy for ischemic stroke with perfusion-imaging selection. $\mathrm{N}$ Engl J Med 372:1009-1018

3. Drouard-De Rousiers E, Lucas L, Richard S et al (2019) Impact of Reperfusion for nonagenarians treated by mechanical thrombectomy: insights from the ETIS registry. Stroke 50:3164-3169

4. Fanaroff AC, Califf RM, Windecker S et al (2019) Levels of evidence supporting American college of cardiology/American heart association and European society of cardiology guidelines, 2008-2018. JAMA 321:1069-1080

5. Goyal M, Demchuk AM, Menon BK et al (2015) Randomized assessment of rapid endovascular treatment of ischemic stroke. $\mathrm{N}$ Engl J Med 372:1019-1030

6. Goyal M, Menon BK, van Zwam WH et al (2016) Endovascular thrombectomy after large-vessel ischaemic stroke: a meta-analysis of individual patient data from five randomised trials. Lancet 387:1723-1731

7. Groot AE, Treurniet KM, Jansen IGH et al (2020) Endovascular treatment in older adults with acute ischemic stroke in the MR CLEAN registry. Neurology 95:e131-e139 
8. Jansen IGH, Mulder M, Goldhoorn RB et al (2018) Endovascular treatment for acute ischaemic stroke in routine clinical practice: prospective, observational cohort study (MR clean registry). BMJ 360:k949

9. Jovin TG, Chamorro A, Cobo E et al (2015) Thrombectomy within 8 hours after symptom onset in ischemic stroke. N Engl J Med 372:2296-2306

10. Mueller-Kronast NH, Zaidat OO, Froehler MT et al (2017) Systematic evaluation of patients treated with neurothrombectomy devices for acute Ischemic stroke: primary results of the stratis registry. Stroke 48:2760-2768

11. Mulder M, Jansen IGH, Goldhoorn RB et al (2018) Time to endovascular treatment and outcome in acute Ischemic stroke: MR clean registry results. Circulation 138:232-240

12. O'leary CP, Cavender MA (2020) Emerging opportunities to harness real world data: an introduction to data sources, concepts, and applications. Diabetes Obes Metab 22(Suppl 3):3-12

13. Powers WJ, Rabinstein AA, Ackerson T et al (2019) Guidelines for the early management of patients with acute Ischemic stroke: 2019 update to the 2018 guidelines for the early management of acute Ischemic stroke: a guideline for Healthcare professionals from the American heartassociation/ American stroke association. Stroke 50:e344-e418

14. Saver JL, Goyal M, Bonafe A et al (2015) Stentretriever thrombectomy after intravenous t-PA vs. t-PA alone in stroke. NEngl J Med 372:2285-2295

15. Saver JL, Goyal M, Van Der Lugt A et al (2016) Time to treatment with endovascular thrombectomy and outcomes from ischemic stroke: a metaanalysis. JAMA 316:1279-1288

16. Stables RH (2002) Observational research in the evidence based environment: eclipsed by the randomised controlled trial? Heart 87:101-102

17. Tiedt S, Bode FJ, Uphaus T et al (2020) Impact of the COVID-19-pandemic on thrombectomy services in Germany. Neurol Res Pract 2:44

18. Turc $G$, Bhogal P, Fischer U et al (2019) European stroke organisation (ESO)-European society for minimally invasive neurological therapy (ESMINT) guidelines on mechanical thrombectomy in acute Ischaemic strokeendorsed by stroke alliance for europe (SAFE). Eur Stroke J 4:6-12

19. Wollenweber FA, Tiedt S, Alegiani A et al (2019) Functional outcome following stroke thrombectomy in clinical practice. Stroke 50:2500-2506

20. Zaidat OO, Bozorgchami H, Ribo M et al (2018) Primary results of the multicenter ARISE II study (analysis of revascularization in Ischemic stroke with embotrap). Stroke 49:1107-1115

21. Zaidat OO, Castonguay AC, Linfante I et al (2018) First pass effect: a new measure for stroke thrombectomy devices. Stroke 49:660-666

22. Zaidat OO, Castonguay AC, Nogueira RG etal (2018) Trevo stent-retriever mechanical thrombectomy for acute ischemic stroke secondary to large vessel occlusion registry. J Neurointerv Surg 10:516-524

23. Zaidat OO, Haussen DC, Hassan AE et al (2019) Impact of etent retriever size on clinical and angiographic outcomes in the stratis stroke thrombectomy registry. Stroke 50:441-447

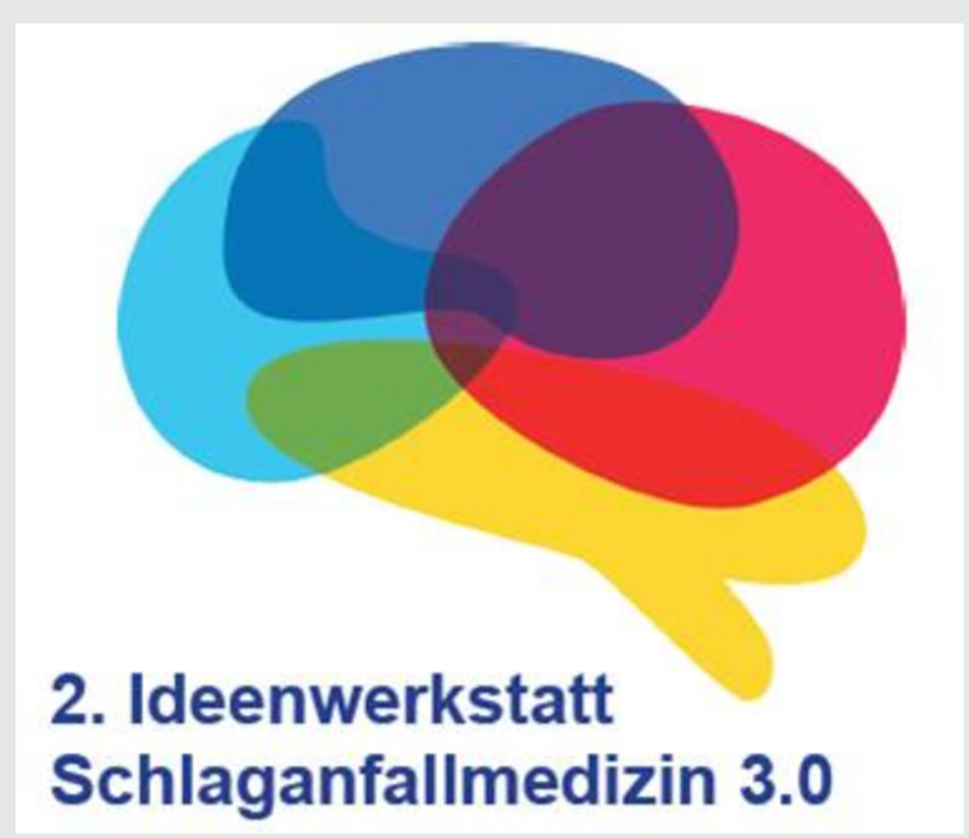

\section{Ideenwerkstatt Schlaganfallmedizin $\mathbf{3 . 0}$}

\section{7.-18. September 2021, Dortmund}

Vom 17. bis 18. September wird in Dortmund die „2. Ideenwerkstatt Schlaganfall 3.0" stattfinden. Die Tagung ist eine Veranstaltung des Neurovaskulären Netz Ruhr e.V. und der MedEconRuhr GmbH, und findet mit Unterstützung der Deutschen Schlaganfall Gesellschaft (DSG), der Stiftung Deutsche Schlaganfall Hilfe (DSH), der AG Nordwestdeutscher Stroke Zirkel e.V. und dem Klinikum Dortmund statt.

Nach langer Pause können wir Sie nun endlich zu einer Präsenzveranstaltung rund um den Schlaganfall einladen - der unmittelbare Kontakt und Austausch ist der entscheidende Faktor dieses Formates.

Stroke Unit, Strukturprüfung, Lysetherapie, NOAKs, multimodale Bildgebung, Thrombektomie, Netzwerkbildung - neue Themen erfordern immer wieder neue Ansätze in der Versorgung von Schlaganfallpatienten, zwingen uns manchmal zum Umdenken oder zum Anpassen von zuvor festgelegten Strategien. Formale Vorgaben erleichtern und erschweren uns dabei manchmal die Arbeit, immer wieder sind praktische Lösungen gefragt um vielfachen Anforderungen gerecht zu werden. Die ist nicht immer einfach.

Mit der Beschreibung der Neuro-vaskulären Netzwerke liegen Zertifizierungsvorgaben vor, die nicht für alle leicht zu erfüllen sind. In der täglichen Praxis werden vielfach Strategien entwickelt, die über das Geforderte hinausgehen oder oft die formalen Vorgaben nicht erfüllen.

Durch die Fokussierung auf die interventionellen Therapien gerät mancherorts das neurologische Kerngeschäft der Stroke Unit Behandlung aus dem Fokus.

Welche Probleme sehen Sie und welche Lösungen haben Sie gefunden? Diese und andere Fragen wollen wir mit Ihnen am 17. und 18. Sept. 2021 bei der Ideenwerksatt Schlaganfall 3.0 diskutieren.

Weitere Informationen zum Programm und zur kostenpflichtigen Anmeldung erhalten Sie unter:

http://nvnr.net; wittmers@medecon.ruhr

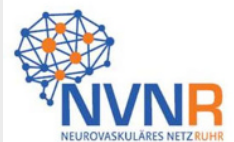

MEDECON nute

Neurovaskuläres Netz Ruhr e.V. 